\title{
An inhomogeneous and anisotropic constitutive model of human dentin
}

\author{
Bo Huo* \\ Institute of Mechanics, Chinese Academy of Science, No. 15 Beisihuanxi Road, Beijing 100080, China
}

Accepted 24 March 2004

\begin{abstract}
Dentin constitutes the major part of human tooth. It is composed of a large number of tubules with both variational radii and radially parallel pattern. In addition, peritubular dentin surrounds each tubule lumen and has a higher elastic modulus than the matrix of dentin, i.e. intertubular dentin. Considering the above microstructural characteristics, a micromechanics model is used in this paper to evaluate the overall elastic properties of dentin. Five independent effective elastic parameters in transverse isotropic elasticity matrix can be expressed analytically by the material parameters of peri- and intertubular dentin and the volume fraction of tubules. To determine the effectivity of this theoretical model, a finite element (FE) model simulating a longitudinal tooth slice in moiré fringe testing of Wang and Weiner (J. Biomech. 31 (1998) 135) was performed. Furthermore, the FE model was developed incorporate modeling of variation of tubule's diameter and softer characteristic of intertubular dentin near the dentin-enamel junction and around the pulp chamber. It turned out that the isoline figure of longitudinal displacement by FE calculation has very similar patterns to the moiré fringe results. However, the FE results of displacement by traditional stress-strain models which regard dentin as a homogeneous and isotropic material show an obviously different strain distributions as compared to published moiré fringes results. Thus the inhomogeneous and anisotropic model developed in this paper more accurately reflects the true physical nature of human dentin.
\end{abstract}

(C) 2004 Elsevier Ltd. All rights reserved.

Keywords: Dentin tubules; Inhomogeneous; Anisotropic; Stress-strain relations; Finite element

\section{Introduction}

In dentin, fluid-filled microscopic tubules extend through the entire dentin from the pulp wall to the dentin-enamel junction (DEJ) or the cementum. In the longitudinal section of dentin (Fig. 1a), the tubules in the root of tooth are approximately parallel, but the tubules in the crown of tooth are radial. In the transverse section (Fig. 1b), the tubules are also radially oriented. The peri- and intertubular dentin represent the mineralized portions in dentin. The peritubular dentin encircles the tubules and is characterized by its high mineral content (Mjör and Fejerskov, 1986). The intertubular dentin occupies the volume outside of peritubular dentin and contains more collagen and less mineral than the peritubular dentin (Fig. 1c).

*Tel.: + 86-106-263-5235; fax: + 86-106-261-3540

E-mail address: huobo@imech.ac.cn (B. Huo).
As dentin is examined closer to the pulp cavity, the diameters of tubules increase gradually, and moreover, the numerary density of tubules also has an increasing trend, although the total number of tubules remain constant (Ketterl, 1961; Tronstad, 1973; Garberoglio and Brännström, 1976; Schilke et al., 2000). The above structural characters of dentin should lead to an inhomogeneous and anisotropic stress-strain relation. In fact, the effects of tubules on the mechanical properties of dentin were considered in the pioneer work of Peyton et al. (1952), but no effects were observed due to the unavailability of micromechanical methods at that time. This led to dentin being treated as a homogeneous and isotropic elastic material.

More recently, several groups have recognized that the presence of dentinal tubules may influence the mechanical properties of dental tissues, e.g. bond strength of restoration (Phrukkanon et al., 1999; Ogata et al., 2001). Microhardness (Pashley et al., 1985; Meredith et al., 1996; Wang and Weiner, 1998) and 


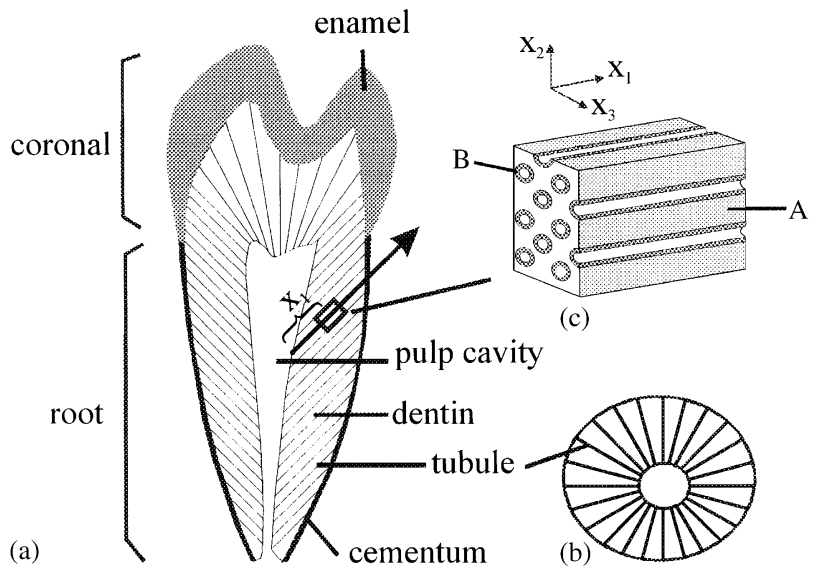

Fig. 1. Schematic figures of a premolar and the microstructure of its dentin: (a) the longitudinal section of the premolar; (b) the transverse section of the dentin; (c) a representative volume element (RVE). Aintertubular dentin, B-peritubular dentin.

nanoindentation (Kinney et al., 1996, 1999) have been adopted to determine the microstructure and physical properties of dentin. Others have reported changes in different chemical components of dentin along the tubules (Wentrup-Byrne et al., 1997).

Furthermore, Kinney et al. (1999) and Huo and Zheng (1999) created micromechanics models of dentin. In Kinney's model, the effects of peri- and intertubular dentin on anisotropic properties of dentin were considered. They measured the elastic modulus of the peritubular dentin as $30 \mathrm{GPa}$ and that of intertubular dentin as $15 \mathrm{GPa}$. They presented a theoretical model in which the tubule lumen lined by a peritubular dentin cuff was assumed to be an isotropic cylindrical inclusion. That assumption may not be appropriate if the composite made up of tubule and peritubular dentin behaves as anisotropic material.

In the authors' previous work (Huo and Zheng, 1999), a varying transverse isotropic stress-strain relation of dentin was established. That theoretical model can reflect the effect of tubules on the inhomogeneous and anisotropic properties of dentin. In that model, however, the peri- and intertubular dentine were regarded as materials with same elastic properties. In this paper the previous model is extended by considering the different mechanical properties of peri- and intertubular dentin, and then a numerical simulation is presented and compared with the previously published moire fringe testing results (Wang and Weiner, 1998).

\section{Materials and methods}

\subsection{Theoretical model}

A representative volume element (RVE) was taken out from the dentin (see Fig. 1c). The RVE was small enough so that the dentin tubules in it could be regarded as being parallel and diameters of the tubules as being invariant. The RVE should also be large enough to contain many tubules so that it can represent the transversely isotropic dentin material in both the local and macroscopic sense. A Cartesian coordinate system $\left\{x_{1}, x_{2}, x_{3}\right\}$ was used, with the $x_{1}$-axis along the dentin tubule direction, the privileged direction of the transverse isotropy.

Using the one-index notations $\tau_{1}=\sigma_{11}, \tau_{4}=\sigma_{23}, \gamma_{2}=$ $\varepsilon_{22}, \gamma_{5}=2 \varepsilon_{31}$, etc., instead of the usual second-order symmetric stress tensor $\sigma_{i j}$ and strain tensor $\varepsilon_{i j}$, the overall elastic stress-strain relationship can be written into the matrix form

$\gamma=\mathbf{D} \tau$,

where the components of elasticity compliance matrix D can be expressed as

$D_{11}=1 / E^{\prime}, \quad D_{22}=D_{33}=1 / E$,

$D_{44}=(1+v) / E, \quad D_{55}=D_{66}=1 / 2 G^{\prime}$,

$D_{12}=D_{21}=D_{13}=D_{31}=-v^{\prime} / E^{\prime}$,

$D_{23}=D_{32}=-v / E$,

other components vanish. The $E, v, E^{\prime}, v^{\prime}$ and $G^{\prime}$ are the five independent effective elastic parameters of the transversely isotropic material. $E^{\prime}$ is the Young's modulus along the direction of tubule. $E$ and $v$ are the Young's modulus and Poisson's ratio within the isotropic plane, respectively. $G^{\prime}$ and $v^{\prime}$ are the shear modulus and Poisson's ratio in the plane of $x_{1} x_{2}$ or $x_{1} x_{3}$, respectively.

Assuming that the peri- and intertubular dentin are homogeneous and isotropic, corresponding elasticities are denoted by $\mathbf{C}^{\mathrm{p}}$ and $\mathbf{C}^{\mathrm{i}}$, Young's moduli by $E_{\mathrm{p}}$ and $E_{\mathrm{i}}$, Poisson's ratios by $v_{\mathrm{p}}$ and $v_{\mathrm{i}}$, respectively. The transverse shapes of tubule and peritubular dentin are supposed to be similar and coaxial circles and their transverse areas within the RVE are denoted by $A_{\mathrm{t}}$ and $A_{\mathrm{p}}$, respectively. The transverse area of the intertubular dentin within the RVE is denoted by $A_{\mathrm{i}}$.

When an ellipsoidal inclusion which contains another ellipsoidal inclusion is embedded in an infinite matrix material, the double-inclusion method can present the overall elasticity of the double inclusions (Nemat-Nasser and Hori, 1993, p. 352). Here a tubule and its surrounding peritubular dentin are considered as the double inclusions, which are assumed to be ellipsoidal with an infinitely long axis parallel to the tubules in RVE. It is considered that in RVE (Fig. 1c) an inclusion (peritubular dentin) including another cavity (dentin tubule) is embedded in matrix material (intertubular dentin). So the overall elasticity of the tubule-peritubule composite is given by

$\mathbf{C}^{\mathrm{tp}}=\mathbf{C}^{\mathrm{i}}[\mathbf{I}+(\mathbf{S}-\mathbf{I}) \mathbf{A}](\mathbf{I}+\mathbf{S A})^{-1}$, 
where the matrix form of Eshelby's tensor $\mathbf{S}$ is given with their components,

$$
\begin{aligned}
& S_{21}=S_{31}=4 v_{\mathrm{i}} / P, \quad S_{23}=S_{32}=\left(4 v_{\mathrm{i}}-1\right) / P, \\
& S_{22}=S_{33}=\left(5-4 v_{\mathrm{i}}\right) / P, \quad S_{44}=2\left(3-4 v_{\mathrm{i}}\right) / P, \\
& S_{55}=S_{66}=4\left(1-v_{\mathrm{i}}\right) / P,
\end{aligned}
$$

other components vanish, in which $P=8\left(1-v_{\mathrm{i}}\right)$. Eshelby's tensor is an important fourth-order tensor in the averaging scheme of micromechanics and it reflects the effect of the eigenstrain within an inclusion with ellipsoidal shape on its surrounding matrix. This tensor depends on the shape of inclusion and the properties of the matrix materials and is independent of the material properties of inclusion. Here the tubule and its surrounding peritubular dentin are assumed to be concentric circles, so their Eshelby's tensors are same.

In (3), $\mathbf{A}$ is defined by

$\mathbf{A}=f_{\mathrm{tp}}(\mathbf{I}-\mathbf{S})^{-1}+\left(1-f_{\mathrm{tp}}\right)\left[\left(\mathbf{C}^{\mathrm{i}}-\mathbf{C}^{\mathrm{p}}\right)^{-1} \mathbf{C}^{\mathrm{i}}-\mathbf{S}\right]^{-1}$.

In the above expression, $f_{\mathrm{tp}}=A_{\mathrm{t}} /\left(A_{\mathrm{t}}+A_{\mathrm{p}}\right)$ and $\mathbf{I}$ denotes the unit matrix. Note that the elasticity of tubules is null in definition (5).

When the elasticity of the matrix deviates considerably from that of inclusion, the two-phase model can be used to evaluate the interaction between inclusions and matrix material (Nemat-Nasser and Hori, 1993, p. 343). In this model, a typical inclusion is embedded in a finite ellipsoidal region with matrix elasticity and then this double-inclusion is embedded in an infinite solid. The volume fraction of the inclusion in that subregion is equal to the actual one of the inclusions in total materials. So the average response of the above subregion can be given to the overall moduli of the two-phase material. Now consider the tubule-peritubule composite as one inclusion with elasticity $\mathbf{C}^{\text {tp }}$ which is embedded in the matrix material of intertubular dentin, then the overall elasticity of dentin can be given as follows:

$$
\begin{aligned}
\mathbf{C}^{\mathrm{d}}= & \mathbf{C}^{\mathrm{i}}\left\{\mathbf{I}+f_{\mathrm{p}}(\mathbf{S}-\mathbf{I})\left[\left(\mathbf{C}^{\mathrm{i}}-\mathbf{C}^{\mathrm{tp}}\right)^{-1} \mathbf{C}^{\mathrm{i}}-\mathbf{S}\right]^{-1}\right\} \\
& \left\{\mathbf{I}+f_{\mathrm{p}} \mathbf{S}\left[\left(\mathbf{C}^{\mathrm{i}}-\mathbf{C}^{\mathrm{tp}}\right)^{-1} \mathbf{C}^{\mathrm{i}}-\mathbf{S}\right]^{-1}\right\}^{-1},
\end{aligned}
$$

where $f_{\mathrm{p}}=\left(A_{\mathrm{t}}+A_{\mathrm{p}}\right) /\left(A_{\mathrm{t}}+A_{\mathrm{p}}+A_{\mathrm{i}}\right)$. So the volume fraction of tubule is $f_{\mathrm{t}}=A_{\mathrm{t}} /\left(A_{\mathrm{t}}+A_{\mathrm{p}}+A_{\mathrm{i}}\right)=f_{\mathrm{tp}} * f_{\mathrm{p}}$.

Substituting (2)-(5) into (6) and solving the following matrix equation:

$\mathbf{D}=\left(\mathbf{C}^{\mathrm{d}}\right)^{-1}$,

the five independent elastic parameters, $E, v, E^{\prime}, v^{\prime}$ and $G^{\prime}$, can be expressed by the material parameters of $E_{\mathrm{p}}$, $E_{\mathrm{i}}, v_{\mathrm{p}}, v_{\mathrm{i}}, f_{\mathrm{tp}}, f_{\mathrm{p}}$. Note that during the above derivations, the inclusions within RVE are implied to be cylindrical, but in fact the volume fractions $f_{\mathrm{tp}}$ and $f_{\mathrm{p}}$ vary with the coordinate $x_{1}$, i.e. $f_{\mathrm{tp}}=f_{\mathrm{tp}}\left(x_{1}\right), f_{\mathrm{p}}=f_{\mathrm{p}}\left(x_{1}\right)$. In this paper, the above expressions for RVE are assumed to still be valid for whole dentin. The overall compliance matrix $\mathbf{D}$ of dentin is dependent on $x_{1}$ and the stress-strain relationship is varying transverse isotropic.

If the peri- and intertubular dentin are regarded as the same material, i.e. $E_{\mathrm{p}}=E_{\mathrm{i}}=E_{\mathrm{m}}, v_{\mathrm{p}}=v_{\mathrm{i}}=v_{\mathrm{m}}, f_{\mathrm{tp}}\left(x_{1}\right)=$ $1, f_{\mathrm{p}}\left(x_{1}\right)=f_{\mathrm{t}}\left(x_{1}\right)=c\left(x_{1}\right)$, the expressions of $E, v, E^{\prime}, v^{\prime}$ and $G^{\prime}$ coincide with the previous results by Huo and Zheng (1999).

\subsection{The establishment of a 2D finite element model}

Wang and Weiner (1998) cut a bucco-lingual section $2 \mathrm{~mm}$ thick out of the central part of a human canine, and applied a compressive force of $500 \mathrm{~N}$ to the top of section. By means of a moiré fringe technique, they presented the displacement distribution, which will be referred to as Wang-Weiner image in this paper, and calculated the strain distribution in the section. As their experiment was simple and was easily simulated, a finite element model was established with the same geometric configuration as their work (Fig. 2a). The DEJ in this FE model had same path as in the model of Wang and Weiner (Fig. 5b). The bottom of the FE model was fixed. A load of magnitude $500 \mathrm{~N}$ was uniformly applied at the top of the model. The FE mesh was generated with 4064 elements and 4183 nodes. All of the preprocessing, calculating and postprocessing were done with the software MSC.Patran 2003.

As the diameter of a human tooth is about $8 \mathrm{~mm}$, a $2 \mathrm{~mm}$ thick slice may be too thick for assuming a 2D structure. However, building a 3D FEM model with inhomogeneous and anisotropic constitutive model requires adequate geometric data in the thickness direction of slice, e.g. the orientation of tubules and the position of DEJ, which entail more assumptions. Considering that the tubules on the front and back
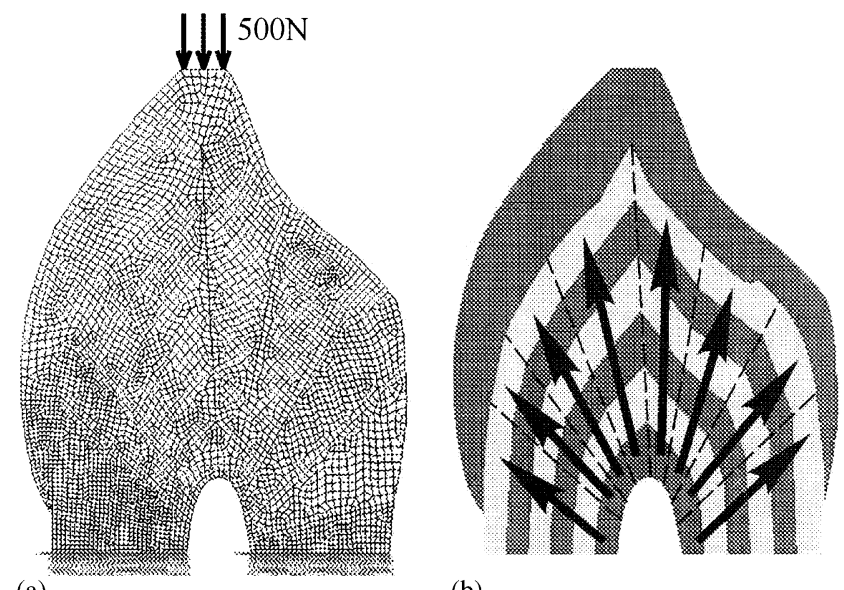

(b)

Fig. 2. (a) The finite element grid and (b) the definitions of inhomogeneous and anisotropic material properties of the section of a human tooth. The dentin is uniformly partitioned into six layers along the tubules. The arrows represent the dentin tubule direction, i.e. the privileged direction of the transverse isotropy. 
planes of slice are symmetrical to the mid-plane and their angle diversity is not very great, it will not affect the computation results greatly to neglect the geometric information in the slice's thickness.

Four FE models with different properties of dentin were established for comparison with Wang-Weiner image. The material properties of enamel for all $\mathrm{FE}$ models were assigned a Young's modulus of $80 \mathrm{GPa}$ and a Poisson's ratio of 0.3 (Rees and Jacobsen, 1997). Most researchers ignore the effect of tubules on the mechanical properties of dentin and regard dentin as homogeneous and isotropic, that model will be called the $H I$ Model in this paper. In the HI Model, the elastic modulus was assigned as $15 \mathrm{GPa}$ and Poisson's ratio as 0.31 (Rees and Jacobsen, 1997).

In the following, it will be described how to establish an inhomogeneous and anisotropic FE model of dentin.

To reflect the variation in mechanical properties, the elements of dentin were equally partitioned into six layers along the tubules and these layers were assigned different material properties according to the above inhomogeneous and anisotropic (IA) stress-strain relation (Fig. 2b).

Fig. 3 shows the curves of three volume fractions, $f_{\mathrm{tp}}\left(x_{1}\right), f_{\mathrm{t}}\left(x_{1}\right)$ and $f_{\mathrm{p}}\left(x_{1}\right)$, varying with the normalized distance, $x_{1} / L$, where $L$ is the length of tubule. Garberoglio and Brännström (1976) measured the diameter and numerical density of tubules in different locations in dentin. Based on their results, the values of $f_{\mathrm{t}}\left(x_{1}\right)$ were calculated. Note that at the wall of the pulp chamber, the value, $f_{\mathrm{t}}(0)=0.32$, was directly measured from the SEM photograph by Garberoglio and Brönnström (1976). Pashley (1989) assumed the area occupied by peritubular dentin as three times that of the tubules, so $f_{\mathrm{p}}\left(x_{1}\right)=4 f_{\mathrm{t}}\left(x_{1}\right)$. Within the predentin, which is close to the pulp cavity, however, there is only intertubular dentin and no peritubular dentin (Mjör and

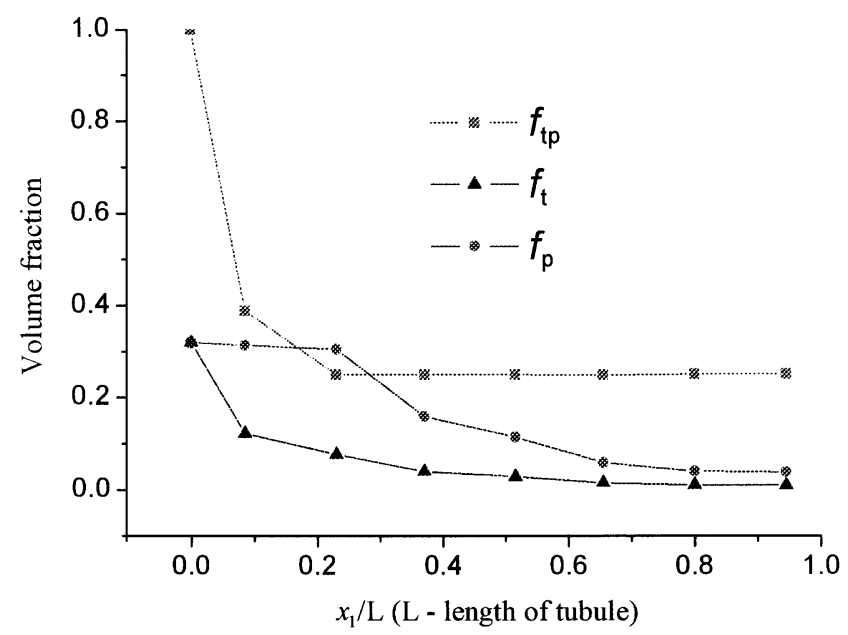

Fig. 3. The curves of three volume fractions $f_{\mathrm{tp}}, f_{\mathrm{t}}$ and $f_{\mathrm{p}}$, varying with the normalized distance $x_{1} / L$ (refer to Garberoglio and Brännström, 1976; Pashley, 1989).
Fejerskov, 1986), i.e. $f_{\mathrm{p}}(0)=f_{\mathrm{t}}(0)$. Therefore, the volume fraction $f_{\mathrm{p}}\left(x_{1}\right)$ may be overestimated when $x_{1}$ approaches zero. Now the area ratio of peritubular dentin was directly measured, i.e. $f_{\mathrm{p}}(0.5 \mathrm{~mm})=0.31$, based on the SEM photograph after decalcification at the position of $0.5 \mathrm{~mm}$ from the pulp wall (Garberoglio and $\mathrm{Br}$ önnström, 1976). The values of $f_{\mathrm{tp}}\left(x_{1}\right)$ can be calculated by the equation, $f_{\mathrm{t}}\left(x_{1}\right)=f_{\mathrm{tp}}\left(x_{1}\right) * f_{\mathrm{p}}\left(x_{1}\right)$.

Kinney et al. (1999) measured the Young's moduli of peri- and intertubular dentin as 30 and $15 \mathrm{GPa}$, respectively. From the nanoindentation testing, Kinney et al. (1996) showed that the intertubular dentin became soft approaching either the pulp cavity or the DEJ, but the hardness of the peritubular dentin remained constant. This led us to assume the Young's modulus of intertubular dentin in layers 1 and 2 towards the pulp cavity as 5 and $10 \mathrm{GPa}$ and in layer 6 near the DEJ as $10 \mathrm{GPa}$, respectively. As there were no experimental values for Poisson's ratio of peri- and intertubular dentin, they were assumed to be 0.3 and 0.4 , respectively. Poisson's ratio of intertubular dentin was assigned the larger value because there are more organic components in it, e.g. collagen fibrils.

The six material parameters of every layer of dentin, $E_{\mathrm{p}}, E_{\mathrm{i}}, v_{\mathrm{p}}, v_{\mathrm{i}}, f_{\mathrm{tp}}, f_{\mathrm{p}}$, could be obtained through the above procedure and then substituted into Eq. (7) to compute the corresponding effective material parameters. This FE model is called IA Model in this paper.

Assuming that the peri- and intertubular dentine were the same matrix material, Huo and Zheng (1999) presented a varying transverse isotropic stress-strain relation of dentin. Based on this theoretical model, the elastic modulus of matrix material was concluded as $29.5 \mathrm{GPa}$ and Poisson's ratio as 0.44 from their experiments (Huo et al., 2000). The effective material parameters of dentin can be calculated by the theoretical model of Huo and Zheng (1999). This FE model is called Huo-Zheng Model in this paper.

Kinney et al. (1999) presented a theoretical model in which the tubule lumen lined by a peritubular dentin cuff was assumed to be an isotropic cylindrical inclusion. In their paper, the elastic modulus of inclusion, $E_{f}$, was calculated as $22.5 \mathrm{GPa}$ and Poisson's ratio, $v_{f}$, was assumed to be 0.25 . The elastic modulus of matrix (i.e. intertubular dentin), $E_{\mathrm{m}}$, was measured as $15 \mathrm{GPa}$ and Poisson's ratio, $v_{\mathrm{m}}$, was assumed to be 0.4 . Substituting the above parameters into their theoretical model, the effective material parameters of dentin can be calculated. This fourth FE model is called the Kinney Model in this paper.

In the above four FE models, the IA Model considered all the features of dentin's mechanical properties based on the presently published experimental data. The Huo-Zheng Model and the Kinney Model were both previously published where the former neglected the difference of peri- and intertubular dentin 

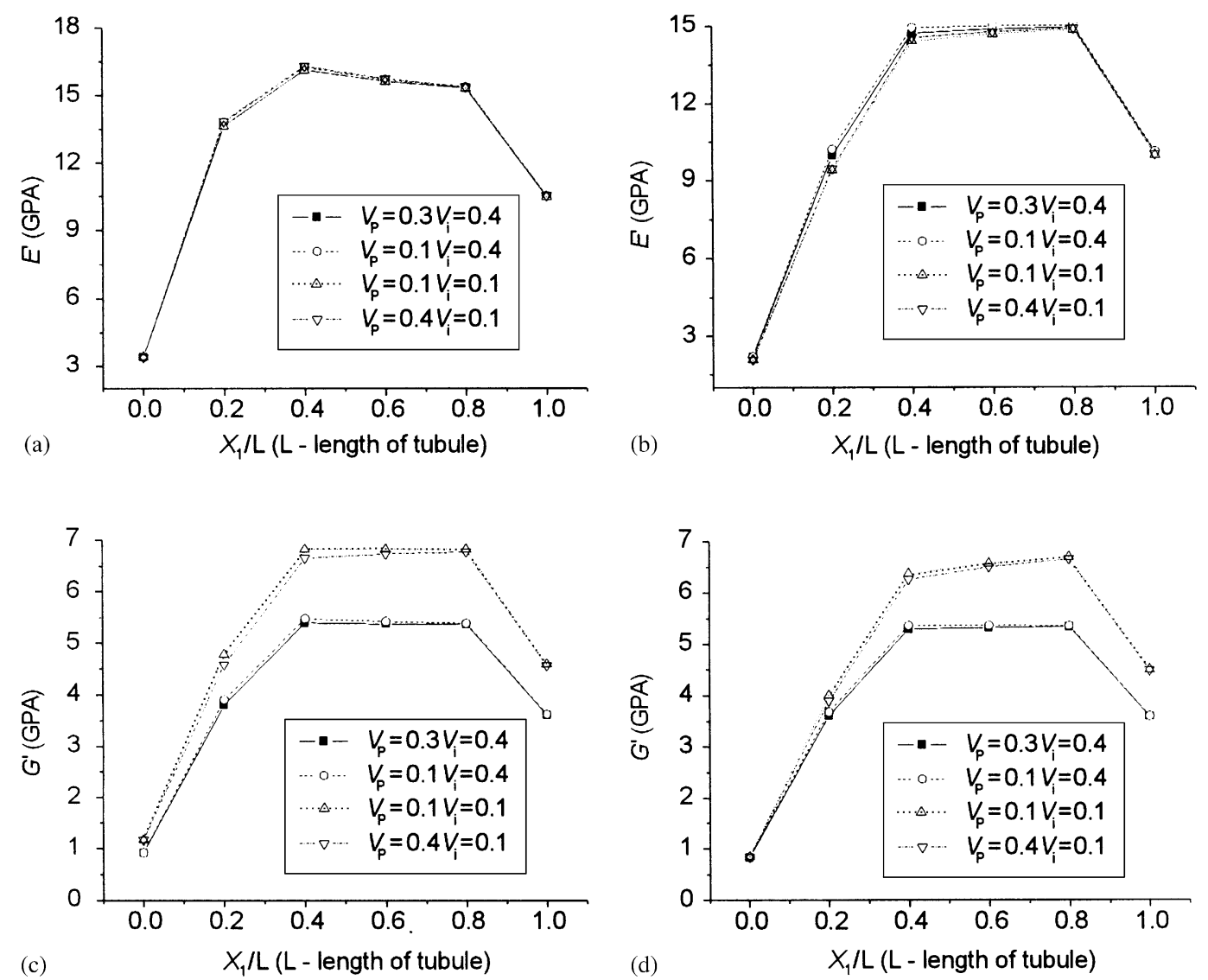

Fig. 4. The effective elastic parameters of dentin at the different normalized distance $x_{1} / L$ for the varying Poisson's ratios of peri- and intertubular dentin, $v_{\mathrm{p}}$ and $v_{\mathrm{i}}$. $E^{\prime}$-Young's modulus in the direction of tubules; $G^{\prime}$ - shear modulus in the plane of $x_{1} x_{2}$ or $x_{1} x_{3}$; $E^{\prime}-$ Young's modulus within the isotropic plane; $G^{\prime}$ - shear modulus within the isotropic plane $(=E /[2(1+v)])$.

and the latter simply assumed the lumen surrounded by peritubular dentin as an isotropic inclusion. Now people usually use the so-called HI Model, which does not consider the microstructure of dentin at all.

\section{Results}

Poisson's ratios of peri- and intertubular dentin, $v_{\mathrm{p}}$ and $v_{\mathrm{i}}$, cannot be measured directly, so its effect on the effective elastic parameters of dentin should be examined. Four pairs of $v_{\mathrm{p}}$ and $v_{\mathrm{i}}$ were presented, i.e. 0.3 and $0.4,0.1$ and $0.4,0.1$ and $0.1,0.4$ and 0.1 . The first pair had been assigned to the IA FE Model. Other pairs represented some combinations of $v_{\mathrm{p}}$ and $v_{\mathrm{i}}$ with larger difference. All of them were substituted into Eq. (7) to calculate the effective elastic parameters of dentin at the different distances from the pulp wall. It can be found that all the elastic parameters have a similar trend, i.e. being lowest near the pulp wall and then increasing up to a platform, finally decreasing near the DEJ (Fig. 4). This variation trend is very similar to the experimental hardness result of Wang and Weiner (1998). It is shown that Young's moduli $E$ and $E^{\prime}$ are negligibly affected by the different cases of Poisson's ratios (Figs. 4a and b).
Little effect of Poisson's ratio $v_{\mathrm{p}}$ of peritubular dentin on shear moduli $G^{\prime}$ and $G(=E /[2(1+v)])$ was found (Figs. $4 \mathrm{c}$ and $\mathrm{d})$. However, the shear moduli obviously change for varying Poisson's ratio $v_{\mathrm{i}}$ of intertubular dentin at some positions with a maximum relative difference of them about $25 \%$. The effective Poisson's ratio $v^{\prime}$ in the plane of $x_{1} x_{2}$ or $x_{1} x_{3}$ is also dominated by $v_{\mathrm{i}}$ and it barely varies at the distance from the pulp wall, which is not shown in the figure.

Fig. 5a is a copy of the fringe pattern of the longitudinal displacement field from the Wang-Weiner image. According to the two FE models, HI Model and IA Model, the isoline images of longitudinal displacement are shown in Figs. $5 \mathrm{~b}$ and c. The corresponding results of two micromechanics model, Huo-Zheng Model and Kinney Model, are presented in Figs. 6a and $b$. To compare the distribution pattern of longitudinal displacement computed by the different models of dentin with the fringe image, the contour lines within the dentin were plotted together (see Figs. 7a and b).

When using the HI Model, it was predicted that the inner part of dentin had the same ability of transferring incisal load as the outer part (Fig. 5b). Thus, the distribution pattern reveals a very flat form in most regions of dentin (Fig. 7a). Only when approaching the 


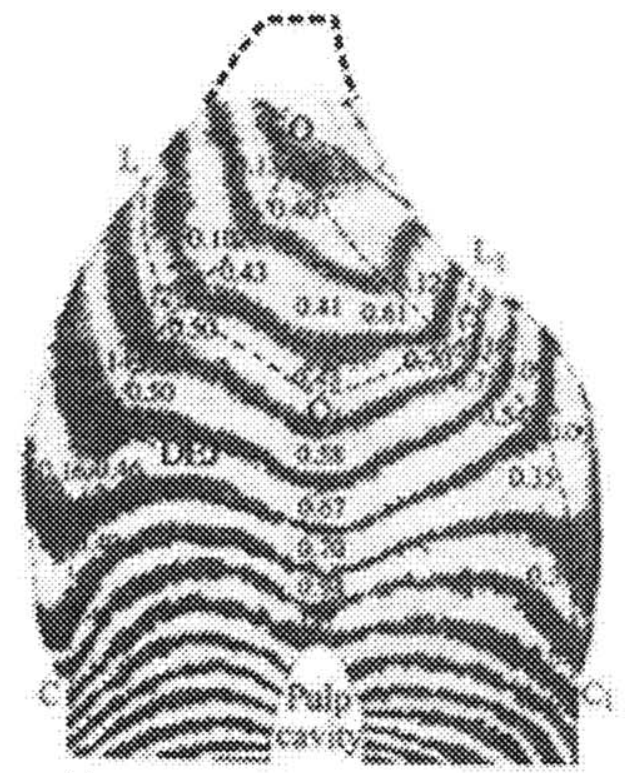

(a)

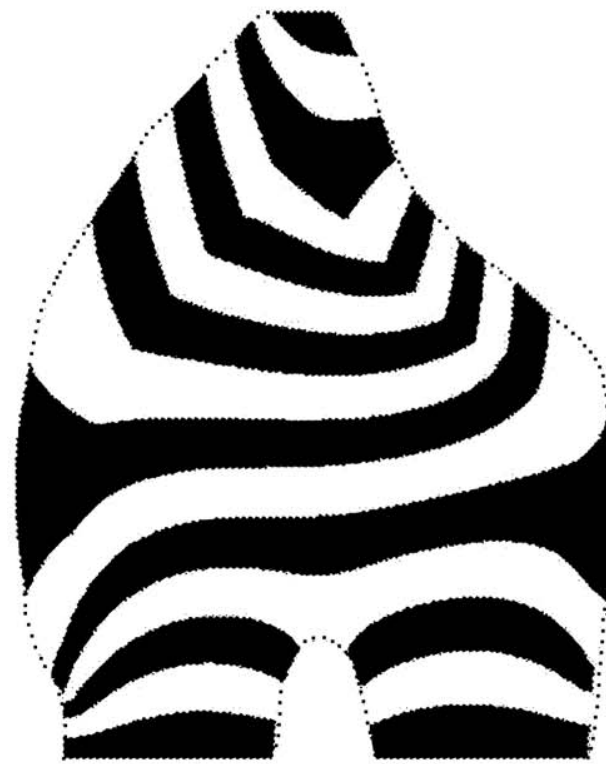

(b)

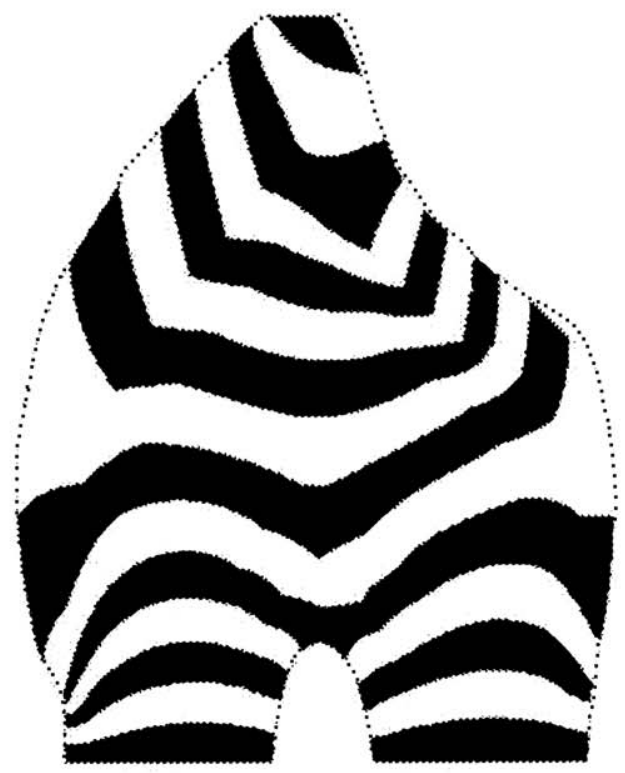

(c)

Fig. 5. (a) The fringe figure of the Wang-Weiner image (Wang and Weiner, 1998). The calculated compressive strain values (\%, the negative sign for the compressive strain was omitted) at different locations of the sample was given by Wang and Weiner (1998). The tip of tooth crown, which was not displayed in the original paper, is redrawn by the dash line. The isoline images of longitudinal displacement are calculated by (b) HI Model and (c) $I A$ Model of dentin.

pulp cavity, is there a slight curving shape for the isoline due to the effect of the pulp chamber.

The IA Model considered the more porous character of dentin near the pulp wall and was constructed so that the intertubular dentin near the DEJ and the pulp wall had softer material properties. Approaching pulp cavity, the IA Model predicted obvious curving patterns for longitudinal displacement isolines within dentin (Fig. 5c). Futhermore, the displacement isolines for the $I A$ Model had a slight turn downwards in the dentin near the DEJ. It can be found that the FE analysis for the $I A$
Model showed very similar fringe patterns to WangWeiner image (Fig. 7a). These characters of isoline pattern were not displayed by the HI Model.

When the stress-strain relation of dentin by Huo and Zheng (1999) was used (Fig. 6a), the displacement field had similar curving patterns to Wang-Weiner image within the center part of dentin (Fig. 7b); but a turn downwards of displacement isolines near the DEJ was not predicted since the softer properties of intertubular dentin near the DEJ was not considered. Being similar to Huo-Zheng Model, the Kinney Model could not 
predict the turn downwards near the DEJ (7b). It was significantly distinguished from Wang-Weiner image in that the longitudinal displacement field of the Kinney Model had a flat form in most regions of dentin.

The above FE results show that the IA Model most closely matched the physical properties of dentin shown by Wang-Weiner image. Other models, such as $H I$, Huo-Zheng and Kinney ones, ignored some features of the mechanical properties of dentin more and less. Therefore, these models gave different patterns of longitudinal displacement from the moiré fringe image.

\section{Discussions}

Two averaging schemes, i.e. double-inclusion method and two-phase model, are used in this paper. An analytical expression of the overall elasticity of dentin is obtained, but the solved expressions of the elastic parameters are too long to be written out integrally in this paper. In fact, the former method is the generalized one of the latter. In the two schemes, the mathematical derivation is exact. Interaction between the inclusions and their immediate surrounding matrix material of
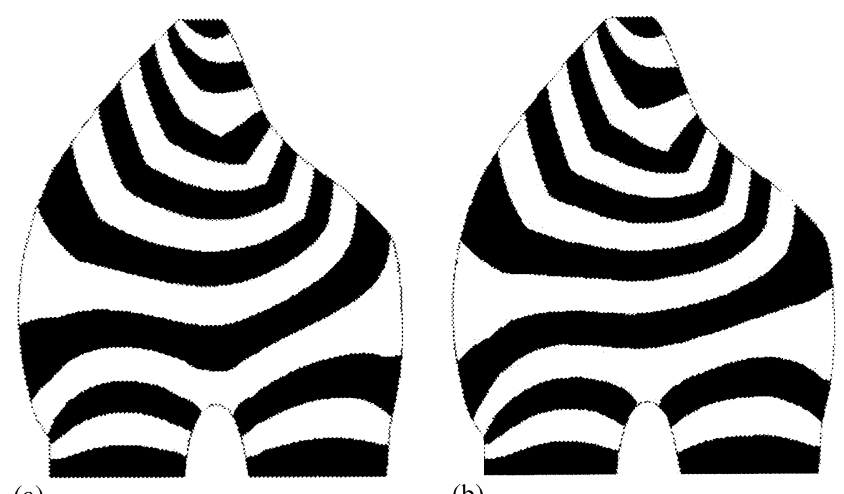

(a)

(b)

Fig. 6. The isoline images of longitudinal displacement are calculated by (a) Huo-Zheng Model and (b) Kinney Model of dentin. different elasticity is directly included in the models (Nemat-Nasser and Hori, 1993, p. 340). The outmost region, however, is assumed to be an infinite matrix material for these two methods, so they may magnify the overall elasticity of the composite when the volume fraction of tubules is large. Therefore, the curvature of displacement isolines close to the pulp cavity in the actual case may be greater than that in the computation results of this paper.

By this inhomogeneous and anisotropic theoretical model of dentin, the FE analysis results of longitudinal displacement were very similar to a previous moiré fringe results in two aspects, i.e. highly curving close to the pulp cavity within the central section of dentin and slightly turning downwards near the DEJ. There are two characters of material properties near the pulp cavity, i.e. being more porous and softer, which contribute to the curving patterns there. Ketterl (1961) measured the value of $76 \%$ for incisors, while Schug-Kösters and Ketterl (1973) obtained a larger value of 79\%. However, several reports gave much smaller volume fractions of $14 \%$ to $32 \%$ (Tronstad, 1973; Garberoglio and Brönnström, 1976; Schilke et al., 2000). The width of collagen and proteoglycan-rich predentin matrix at the boundary of the pulp chamber is thought to be about $50 \mu \mathrm{m}$ thick. So it is believed that even if there are higher volume fractions of tubules near the pulp chamber, their influence fall off rapidly away from the pulp wall, due to the need of enduring the occlusal loading. When establishing the IA FE Model, the elastic modulus of intertubular dentin was defined as smaller values, up to $5 \mathrm{GPa}$ close to the pulp wall and $10 \mathrm{GPa}$ near the DEJ. The softer material properties of intertubular dentin in the above regions contribute to the curving patterns near the pulp cavity and lead to turning downwards of displacement isolines near the DEJ. When the softer properties of peri- and intertubular dentin were ignored, i.e. $E_{\mathrm{p}}$ and $E_{\mathrm{i}}$ were assumed to be constant, a turn downwards of displacement isolines near the DEJ disappeared and the curvatures of them towards the

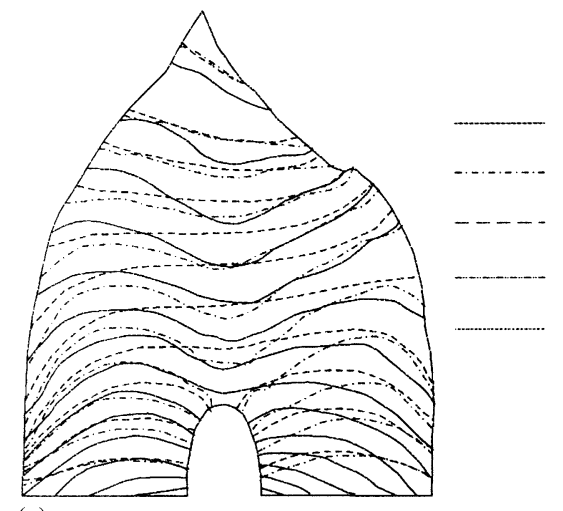

(a)

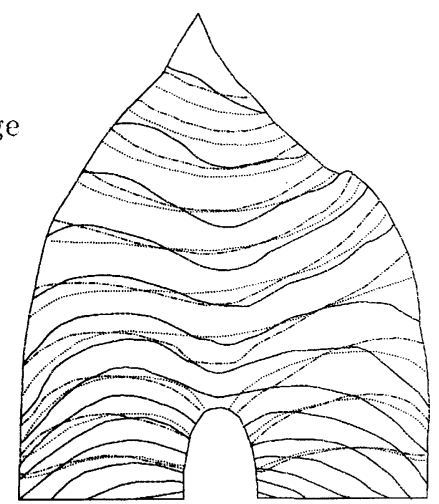

(b) Wang-Weiner image IA model HI model Huo-Zheng mode1 Kinney model

Fig. 7. The comparison of the contour lines of longitudinal displacement within the dentin computed by different FE models with the ones obtained from the Wang-Weiner image. 
pulp cavity became less. So the inhomogeneity has greater impact to the distribution pattern than the anisotropy (also refer to Huo and Zheng, 2002).

The experimental data for the Young's moduli of peri- and intertubular dentin along the thickness of dentin are still imperfect. Some macroscopic tests had been done to study the hardness of dentin from the DEJ towards the pulp cavity (Smith and Cooper, 1971; Pashley et al., 1985; Watanabe et al., 1996; Lertchirakarn et al., 2001). Using these corresponding methods, the mechanical properties of peri- and intertubular dentin could not be separately determined. Owing to the development of atomic force microscopy fitted with a diamond stylus, however, one can look at the mechanical properties of intertubular dentin separated from peritubular dentin. Some studies showed that the Young's modulus and hardness of peritubular dentin did not vary with depth and the hardness of intertubular dentin in DEJ was higher than near the pulp (Kinney et al., 1999; Marshall et al., 2001). But more detailed testing for the Young's moduli along the tubule's direction may still be necessary.

Poisson's ratios of peri- and intertubular dentin have not yet been experimentally determined because of their small size, but an experiment may be designed by the theoretical model developed in this paper. For example, a small cube extracted from dentin can be compressed parallel and perpendicular to the tubule directions by a specially modified atomic-force microscope and the corresponding load-displacement measurements can be easily obtained. This would permit two Poisson's ratios to be obtained by solving the two parameter equations along the directions parallel and perpendicular to the tubule. The use of accurate Poisson's ratio would improve the FE simulations.

\section{Acknowledgements}

The author thanks Prof. Q.S. Zheng for the helpful discussions about the theoretical model and also thanks Prof. D.H. Pashley and Dr. Z.M. He for the kind syntax improvement and the helpful discussions on the results. This research was supported by the National Natural Science Foundation of China $(19525207,30300076)$ and the Tsinghua University Fundamental Research Foundation (Jc1999033).

\section{References}

Garberoglio, R., Brännström, M., 1976. Scanning electron microscopic investigation. Archives of Oral Biology 21, 355-362.

Huo, B., Zheng, Q.S., 1999. Effect of dentin tubules to the mechanical properties of dentin. Part I: Stress-strain relations and strength criterion. Acta Mechanica Sinica (English series) 15 (4), 355-364.
Huo, B., Zheng, Q.S., 2002. Effect of dentin tubules to the mechanical properties of dentin. Part III: Numerical analysis. Acta Mechanica Sinica (English series) 18 (6), 629-637.

Huo, B., Zheng, Q.S., Zhang, Q., Wang, J.D., 2000. Effect of dentin tubules to the mechanical properties of dentin. Part II: Experimental study. Acta Mechanica Sinica (English series) 16 (1), 75-82.

Ketterl, W., 1961. The dentin in permanent human teeth. Stoma 14, 79-96.

Kinney, J.H., Balooch, M., Marshall, S.J., Marshall, W.J.R., Weih, T.P., 1996. Hardness and Young's modulus of human peritubular and intertubular dentine. Archives of Oral Biology 41 (1), 9-13.

Kinney, J.H., Balooch, M., Marshall, G.W., Marshall, S.J., 1999. A micromechanics model of the elastic properties of human dentine. Archives of Oral Biology 44, 813-822.

Lertchirakarn, V., Palamara, E.A., Messer, H.H., 2001. Anisotrophy of tensile strength of root dentin. Journal of Dental Research 80, 453-456.

Marshall, G.W., Habelitz, S., Gallagher, R., Balooch, M., Balooch, G., Marshall, S.J., 2001. Nanomechanical properties of hydrated carious human dentin. Journal of Dental Research 80, 1768-1771.

Meredith, N., Sherriff, M., Setchell, D.J., Swanson, S.A.V., 1996. Measurement of the microhardness and Young's modulus of human enamel and dentine using an indentation technique. Archives of Oral Biology 41, 539-545.

Mjör, I.A., Fejerskov, O., 1986. Human Oral Embryology and Histology. Munksgaard, Copenhagen.

Nemat-Nasser, S., Hori, M., 1993. Micromechanics: Overall Properties of Heterogeneous Materials. Elsevier Science Publishers B.V., Amsterdam.

Ogata, M., Okuda, M., Nakajima, M., Pereira, P.N.R., Sano, H., Tagami, J., 2001. Influence of the direction of tubules on bond strength to dentin. Operative Dentistry 26, 27-35.

Pashley, D., 1989. Dentin: a dynamic substrate - a review. Scanning Microscopy 3, 161-176.

Pashley, D., Okabe, A., Parham, P., 1985. The relationship between dentin microhardness and tubule density. Endodontics and Dental Traumatology 1, 176-179.

Peyton, F.A., Mahier, D.B., Hershenov, B., 1952. Physical properties of dentine. Journal of Dental Research 31, 366-370.

Phrukkanon, S., Burrow, M.F., Tyas, M.J., 1999. The effect of dentine location and tubule orientation on the bond strengths between resin and dentine. Journal of Dentistry 27, 265-274.

Rees, J.S., Jacobsen, P.H., 1997. Elastic modulus of the periodontal ligament. Biomaterials 18, 995-999.

Schilke, R., Lisson, J., Baub, O., Geurtsen, W., 2000. Comparison of the number and diameter of dentinal tubules in human and bovine dentine by scanning electron microscopic investigation. Archives of Oral Biology 45, 355-361.

Schug-Kösters, M., Ketterl, W., 1973. Pulpenerkrankungen im Milchgebib und ihre Folgezusände einschlieblich ihrer Behandlung. In: Ericenbach. E. (Ed.), Kinderzahn-heikunde im Vorschulalter, Barth, Leipzig.

Smith, D.C., Cooper, W.E.G., 1971. The determination of shear strength: a method using a micro-punch apparatus. British Dental Journal 130, 333-337.

Tronstad, L., 1973. Ultrastructure observations on human coronal dentin. Scandinavian Journal of Dental Research 81, 101-111.

Wang, R.Z., Weiner, S., 1998. Strain-structure relations in human teeth using moiré fringes. Journal of Biomechanics 31, 135-141.

Watanabe, L.G., Marshall, G.W., Marshall, S.J., 1996. Dentin shear strength, effect of tubule orientation and intratooth location. Dental Materials 12, 109-115.

Wentrup-Byrne, E., Armstrong, C.A., Armstrong, R.S., Collins, M., 1997. Fourier transform Raman microscopic mapping of the molecular components in a human tooth. Journal of Raman Spectroscopy 28, 151-158. 